Preprint of the paper

"A Boundary Element Formulation for the Substation Grounding Design" I. Colominas, F. Navarrina, M. Casteleiro (1999)

Advances in Engineering Software Journal, 30 , 603--700.

http://caminos.udc.es/gmni 


\title{
A BOUNDARY ELEMENT FORMULATION FOR THE SUBSTATION GROUNDING DESIGN
}

I. Colominas, F. Navarrina, and M. Casteleiro

E.T.S. de Ingenieros de Caminos, Canales y Puertos. Dpto. de Métodos Matemáticos y Representación

Universidad de La Coruña

Campus de Elviña. 15192-La Coruña, SPAIN

Tel: (+34).981.16.70.00; Fax: (+34).981.16.71.70

E-mail: colominas@iccp.udc.es, navarrina@iccp.udc.es, casteleiro@udc.es

\begin{abstract}
A Boundary Element approach for the numerical computation of substation grounding systems is presented. In this general formulation, several widespread intuitive methods (such as Average Potential Method) can be identified as the result of specific choices for the test and trial functions and suitable assumptions introduced in the BEM formulation to reduce computational cost. While linear and parabolic leakage current elements allow to increase accuracy, computing time is drastically reduced by means of new completely analytical integration techniques and semi-iterative methods for solving linear equations systems. This BEM formulation has been implemented in a specific Computer Aided Design system for grounding analysis developed in the last years. The feasibility of this new approach is demonstrated with its application to a real problem.
\end{abstract}

KEY WORDS : Earthing Computation, Boundary Element Methods, Grounding Grid Design

\section{Introduction}

In general, a safe earthing system has the objectives of granting the integrity of equipments and the continuity of the service under fault conditions - providing means to carry and dissipate electric currents into the ground - and safeguarding that a person working or walking in the surroundings of grounded installations is not exposed to the danger of suffering an electrical shock. To achieve these goals, the equivalent electrical resistance of the system must be low enough to assure that fault currents dissipate mainly through the grounding grid into the earth, while maximum potential gradients between close points on the earth surface must be kept under certain tolerances (step, touch and mesh voltages) [1,2].

Physical phenomena underlying fault currents dissipation into the earth can be modelled by means of Maxwell's Electromagnetic Theory [3]. Constraining the analysis to obtain the electrokinetic steady-state response and neglecting the inner resistivity of the earthing conductors - therefore, potential can be assumed constant in every point of the electrodes surface-, the $3 \mathrm{D}$ problem associated with an electrical current derivation to earth can be written as

$$
\begin{gathered}
\boldsymbol{\sigma}=-\boldsymbol{\gamma} \operatorname{grad}(V), \quad \operatorname{div}(\boldsymbol{\sigma})=0 \text { in } E, \\
\boldsymbol{\sigma}^{t} \boldsymbol{n}_{E}=0 \quad \text { in } \Gamma_{E}, \quad V=V_{\Gamma} \quad \text { in } \quad \Gamma, \quad V \longrightarrow 0 \quad \text { if }|\boldsymbol{x}| \rightarrow \infty,
\end{gathered}
$$

where $E$ is the earth, $\boldsymbol{\gamma}$ its conductivity tensor, $\Gamma_{E}$ the earth surface, $\boldsymbol{n}_{E}$ its normal exterior unit field and $\Gamma$ the electrode surface $[4,5]$. The solution at this problem gives the potential $V$ and the current density $\boldsymbol{\sigma}$ 
at an arbitrary point $\boldsymbol{x}$ when the electrode attains a voltage $V_{\Gamma}$ (Ground Potential Rise or GPR) relative to a distant grounding point assumed to be at the potential of remote earth. Since $V$ and $\boldsymbol{\sigma}$ are proportional to the GPR value, the normalized boundary condition $V_{\Gamma}=1$ is not restrictive at all.

On the other hand, the leakage current density $\sigma$ at an arbitrary point of the earthing electrode surface, the total surge current $I_{\Gamma}$ leaked into the ground when fault conditions occur, and the equivalent resistance of the earthing system $R_{e q}$ (apparent resistance of the earth-electrode circuit) can be written as:

$$
\sigma=\boldsymbol{\sigma}^{t} \boldsymbol{n}, \quad I_{\Gamma}=\iint_{\Gamma} \sigma d \Gamma, \quad R_{e q}=\frac{V_{\Gamma}}{I_{\Gamma}}
$$

being $\boldsymbol{n}$ the normal exterior unit field to $\Gamma$.

For practical purposes, the hypothesis of homogeneous and isotropic soil can be considered acceptable [2], and its conductivity tensor $\boldsymbol{\gamma}$ can be substituted by a meassured apparent scalar conductivity $\gamma$. Otherwise, since the kind of techniques presented in this paper can be extended to multi-layer soil models (these models represent the ground stratified into two or more layers of appropriate thickness each one with a different value of $\gamma[6]$ ), further discussion and examples are restricted to uniform soils. If one further assumes that the earth surface is horizontal, symmetry allows to rewrite (1) in terms of a Dirichlet Exterior Problem [5]. In practice, the particular geometry of the earthing electrode in most electrical installations —a grid of interconnected bare cylindrical conductors, horizontally buried and supplemented by a number of vertical rods, which ratio diameter/lenght uses to be relatively small (of the order of $10^{-3}$ ) - makes very difficult to obtain analytical solutions to this kind of problems. Therefore, the use of standard numerical techniques (such as Finite Differences or Finite Elements) requires the discretization of domain $E$, and to obtain sufficiently accurate results should imply unacceptable computing efforts in memory storage and CPU time.

On the other hand, since computation of potential is only required on the earth surface $\Gamma_{E}$, and the equivalent resistance can be easily obtained in terms of the leakage current density at points of the earthing electrode surface (2), a Boundary Element approach (which would only require the discretization of the grounding surface $\Gamma$ ) seems to be the right choice $[7,8,9]$.

\section{General Boundary Element Formulation}

The application of results of the Potential Theory to problem (1) allows to express the potential $V$ at an arbitrary point $\boldsymbol{x}$ on the earth $E$ in terms of the unknown leakage current density $\sigma$ in $\Gamma$, in the integral form:

$$
V(\boldsymbol{x})=\frac{1}{4 \pi \gamma} \iint_{\boldsymbol{\xi} \in \Gamma} k(\boldsymbol{x}, \boldsymbol{\xi}) \sigma(\boldsymbol{\xi}) d \Gamma
$$

with the weakly singular kernel $k(\boldsymbol{x}, \boldsymbol{\xi})$

$$
k(\boldsymbol{x}, \boldsymbol{\xi})=\left(\frac{1}{r(\boldsymbol{x}, \boldsymbol{\xi})}+\frac{1}{r\left(\boldsymbol{x}, \boldsymbol{\xi}^{\prime}\right)}\right), \quad r(\boldsymbol{x}, \boldsymbol{\xi})=|\boldsymbol{x}-\boldsymbol{\xi}|,
$$

where $\boldsymbol{\xi}^{\prime}$ is the symmetric of $\boldsymbol{\xi}$ with respect to the earth surface $[4,5,10,11]$. 
Since (3) holds on the earthing electrode surface $\Gamma$, the boundary condition $V_{\Gamma}=1$ leads to a Fredholm integral equation of the first kind on $\Gamma$ with quasi-singular kernel (4), which solution is the unknown leakage current density $\sigma[5]$. Moreover, the variational form

$$
\iint_{\boldsymbol{\chi} \in \Gamma} w(\boldsymbol{\chi})(V(\boldsymbol{\chi})-1) d \Gamma=0
$$

must be satisfied for all members $w(\boldsymbol{\chi})$ of a suitable class of test functions defined on $\Gamma$.

Now, for a given set of $\mathcal{N}$ trial functions $\left\{N_{i}(\boldsymbol{\xi})\right\}$ defined on $\Gamma$, and for a given set of $\mathcal{M} 2 \mathrm{D}$ boundary elements $\left\{\Gamma^{\alpha}\right\}$, the unknown leakage current density $\sigma$ and the earthing electrode surface $\Gamma$ can be discretized in the form

$$
\sigma(\boldsymbol{\xi})=\sum_{i=1}^{\mathcal{N}} \sigma_{i} N_{i}(\boldsymbol{\xi}), \quad \Gamma=\bigcup_{\alpha=1}^{\mathcal{M}} \Gamma^{\alpha},
$$

and a discretized form of potential (3) can be written as

$$
V(\boldsymbol{x})=\sum_{i=1}^{\mathcal{N}} \sigma_{i} V_{i}(\boldsymbol{x}), \quad V_{i}(\boldsymbol{x})=\sum_{\alpha=1}^{\mathcal{M}} V_{i}^{\alpha}(\boldsymbol{x}),
$$

being $V_{i}^{\alpha}(\boldsymbol{x})$ potential coefficients

$$
V_{i}^{\alpha}(\boldsymbol{x})=\frac{1}{4 \pi \gamma} \iint_{\boldsymbol{\xi} \in \Gamma^{\alpha}} k(\boldsymbol{x}, \boldsymbol{\xi}) N_{i}(\boldsymbol{\xi}) d \Gamma^{\alpha}
$$

Then, for a given set of $\mathcal{N}$ test functions $\left\{w_{j}(\chi)\right\}$ defined on $\Gamma$, the variational statement (5) is reduced to the system of linear equations

$$
\begin{gathered}
\sum_{i=1}^{\mathcal{N}} R_{j i} \sigma_{i}=\nu_{j}, \quad j=1, \ldots, \mathcal{N} ; \\
R_{j i}=\sum_{\beta=1}^{\mathcal{M}} \sum_{\alpha=1}^{\mathcal{M}} R_{j i}^{\beta \alpha}, \quad \nu_{j}=\sum_{\beta=1}^{\mathcal{M}} \nu_{j}^{\beta} ; \\
R_{j i}^{\beta \alpha}=\frac{1}{4 \pi \gamma} \iint_{\boldsymbol{\chi} \in \Gamma^{\beta}} w_{j}(\boldsymbol{\chi}) \iint_{\boldsymbol{\xi} \in \Gamma^{\alpha}} k(\boldsymbol{\chi}, \boldsymbol{\xi}) N_{i}(\boldsymbol{\xi}) d \Gamma^{\alpha} d \Gamma^{\beta} \\
\nu_{j}^{\beta}=\iint_{\boldsymbol{\chi} \in \Gamma^{\beta}} w_{j}(\boldsymbol{\chi}) d \Gamma^{\beta} .
\end{gathered}
$$

In practice, the number of $2 \mathrm{D}$ discretizations required to solve the above stated equations in real problems implies an extremely large number of degrees of freedom. Moreover, coefficients matrix in (9) is full and the computation of each term requires double integration on a $2 \mathrm{D}$ domain, and therefore some additional simplifications must be introduced to overcome the problem complexity. 


\section{Aproximated 1D Variational Statement}

With this scope, it is possible to introduce in our statement one of the hypotheses widely used in most of the practical methods related in the literature [1,2,11]. Thus, taking into account the real geometry of grounding grids in practice, it seems reasonable to consider that the leakage current density is constant around the cross section of the cylindrical electrode $[4,5]$.

Hence, if we denote $L$ the whole set of axial lines of the buried conductors, $\widehat{\boldsymbol{\xi}}$ the orthogonal projection over the bar axis of a given generic point $\boldsymbol{\xi} \in \Gamma, \phi(\widehat{\boldsymbol{\xi}})$ the electrode diameter, $C(\widehat{\boldsymbol{\xi}})$ the circumferential perimeter of the cross section at $\widehat{\boldsymbol{\xi}}$, and $\widehat{\sigma}(\widehat{\boldsymbol{\xi}})$ the approximated leakage current density at this point (assumed uniform around the cross section), equation (3) can be written in the form

$$
\hat{V}(\boldsymbol{x})=\frac{1}{4 \pi \gamma} \int_{\widehat{\boldsymbol{\xi}} \in L}\left[\int_{\boldsymbol{\xi} \in C(\widehat{\boldsymbol{\xi}})} k(\boldsymbol{x}, \boldsymbol{\xi}) d C\right] \widehat{\sigma}(\widehat{\boldsymbol{\xi}}) d L
$$

This assumption of circumferential uniformity seems to be quite adecquate and not too restrictive due to the specific geometry of these earthing electrodes in real cases. Nevertheless, because the leakage current is not really uniform around the cross section, boundary condition $V_{\Gamma}=1$ can not be exactly satisfied now at every point on the electrode surface and variational equality (5) does not hold anymore. Therefore, if we restrict the class of trial functions to those with circumferential uniformity, that is $w(\boldsymbol{\chi})=\widehat{w}(\widehat{\boldsymbol{\chi}}) \forall \boldsymbol{\chi} \in C(\widehat{\chi})$, (5) results:

$$
\int_{\widehat{\boldsymbol{\chi}} \in L} \widehat{w}(\widehat{\boldsymbol{\chi}})\left[\pi \phi(\hat{\boldsymbol{\chi}})-\frac{1}{4 \pi \gamma} \int_{\hat{\boldsymbol{\xi}} \in L} K(\hat{\boldsymbol{\chi}}, \widehat{\boldsymbol{\xi}}) \hat{\sigma}(\widehat{\boldsymbol{\xi}}) d L\right] d L=0
$$

for all members $\widehat{w}(\widehat{\chi})$ of a suitable class of test functions defined on $L$, being $K(\widehat{\boldsymbol{\chi}}, \widehat{\boldsymbol{\xi}})$ the integral kernel

$$
K(\widehat{\boldsymbol{\chi}}, \widehat{\boldsymbol{\xi}})=\int_{\boldsymbol{\chi} \in C(\hat{\boldsymbol{\chi}})}\left[\int_{\boldsymbol{\xi} \in C(\widehat{\boldsymbol{\xi}})} k(\boldsymbol{\chi}, \boldsymbol{\xi}) d C\right] d C
$$

Resolution of integral equation (14) involves discretization of the domain -in this case, the whole set of axial lines of the buried conductors $L-$. Thus, for given sets of $n$ trial functions $\left\{\widehat{N}_{i}(\widehat{\boldsymbol{\xi}})\right\}$ defined on $L$ and $m$ 1D boundary elements $\left\{L^{\alpha}\right\}$, the unknown approximated leakage current density $\hat{\sigma}$ and the whole set of axial lines of the buried conductors $L$ can be discretized in the form

$$
\widehat{\sigma}(\widehat{\boldsymbol{\xi}})=\sum_{i=1}^{n} \widehat{\sigma}_{i} \widehat{N}_{i}(\widehat{\boldsymbol{\xi}}), \quad L=\bigcup_{\alpha=1}^{m} L^{\alpha}
$$

In these terms, a discretized version of the aproximated potential (13) can be obtained as

$$
\widehat{V}(\boldsymbol{x})=\sum_{i=1}^{n} \widehat{\sigma}_{i} \hat{V}_{i}(\boldsymbol{x}), \quad \widehat{V}_{i}(\boldsymbol{x})=\sum_{\alpha=1}^{m} \widehat{V}_{i}^{\alpha}(\boldsymbol{x}),
$$




$$
\widehat{V}_{i}^{\alpha}(\boldsymbol{x})=\frac{1}{4 \pi \gamma} \int_{{\widehat{\boldsymbol{\xi}} \in L^{\alpha}}}\left[\int_{\boldsymbol{\xi} \in C(\widehat{\boldsymbol{\xi}})} k(\boldsymbol{x}, \boldsymbol{\xi}) d C\right] \widehat{N}_{i}(\widehat{\boldsymbol{\xi}}) d L
$$

On the other hand, for a suitable selection of $n$ test functions $\left\{\widehat{w}_{j}(\widehat{\boldsymbol{\chi}})\right\}$ defined on $L$, variational statement (14) is reduced to the system of linear equations

$$
\begin{gathered}
\sum_{i=1}^{n} \widehat{R}_{j i} \widehat{\sigma}_{i}=\widehat{\nu}_{j}, \quad j=1, \ldots, n ; \\
\widehat{R}_{j i}=\sum_{\beta=1}^{m} \sum_{\alpha=1}^{m} \widehat{R}_{j i}^{\beta \alpha}, \quad \widehat{\nu}_{j}=\sum_{\beta=1}^{m} \widehat{\nu}_{j}^{\beta} ;
\end{gathered}
$$

where $\widehat{R}_{j i}^{\beta \alpha}$ and $\widehat{\nu}_{j}^{\beta}$ coefficients can be obtained as

$$
\begin{gathered}
\widehat{R}_{j i}^{\beta \alpha}=\frac{1}{4 \pi \gamma} \int_{\widehat{\boldsymbol{\chi}} \in L^{\beta}} \widehat{w}_{j}(\hat{\boldsymbol{\chi}})\left[\int_{\hat{\boldsymbol{\xi}}_{\in L^{\alpha}}} K(\hat{\boldsymbol{\chi}}, \widehat{\boldsymbol{\xi}}) \widehat{N}_{i}(\widehat{\boldsymbol{\xi}}) d L\right] d L, \\
\widehat{\nu}_{j}^{\beta}=\int_{\widehat{\boldsymbol{\chi}} \in L^{\beta}} \pi \phi(\widehat{\boldsymbol{\chi}}) \widehat{w}_{j}(\hat{\boldsymbol{\chi}}) d L .
\end{gathered}
$$

On a regular basis, the computational work required to solve a real problem is drastically reduced by means of this $1 \mathrm{D}$ formulation with respect to the one given by expressions (9), (10), (11) and (12), because integrals on the circumferential perimeter of electrodes are taken apart of integrals on their axial lines. However, extensive computing is still required, mainly for circumferential integration in (18) and (21), and further simplifications are necessary to reduce computing time under acceptable levels [5].

\subsection{Simplified 1D Boundary Element Formulation}

The inner integral of kernel $k(\boldsymbol{x}, \boldsymbol{\xi})$ in $(18)$ can be written as sum of two terms:

$$
\int_{\boldsymbol{\xi} \in C(\hat{\boldsymbol{\xi}})} k(x, \boldsymbol{\xi}) d C=\int_{\boldsymbol{\xi} \in C(\hat{\boldsymbol{\xi}})} \frac{d C}{r(\boldsymbol{x}, \boldsymbol{\xi})}+\int_{\boldsymbol{\xi} \in C(\widehat{\boldsymbol{\xi}})} \frac{d C}{r\left(x, \boldsymbol{\xi}^{\prime}\right)}
$$

Analyzing the first of them, distance $r(\boldsymbol{x}, \boldsymbol{\xi})$ between any point $\boldsymbol{x}$ of the domain and any point $\boldsymbol{\xi}$ at the earthing electrode surface can be expressed as:

$$
r(\boldsymbol{x}, \boldsymbol{\xi})=\sqrt{|\boldsymbol{x}-\widehat{\boldsymbol{\xi}}|^{2}+\frac{\phi^{2}(\widehat{\boldsymbol{\xi}})}{4}-|\boldsymbol{x}-\widehat{\boldsymbol{\xi}}| \phi(\widehat{\boldsymbol{\xi}}) \sin \omega \cos \theta}
$$

where $\theta$ is the angular position in the perimeter of cross section of the cylindrical conductor, and $\omega$ is the angle formed by the vector that links $\boldsymbol{x}$ with its projection $\widehat{\boldsymbol{\xi}}(\widehat{\boldsymbol{\xi}}-\boldsymbol{x})$ and the unit vector of bar axis $\widehat{\boldsymbol{s}}(\widehat{\boldsymbol{\xi}})$, that is

$$
\sin \omega=\frac{|(\widehat{\boldsymbol{\xi}}-\boldsymbol{x}) \times \widehat{\boldsymbol{s}}(\widehat{\boldsymbol{\xi}})|}{|\widehat{\boldsymbol{\xi}}-\boldsymbol{x}|}
$$




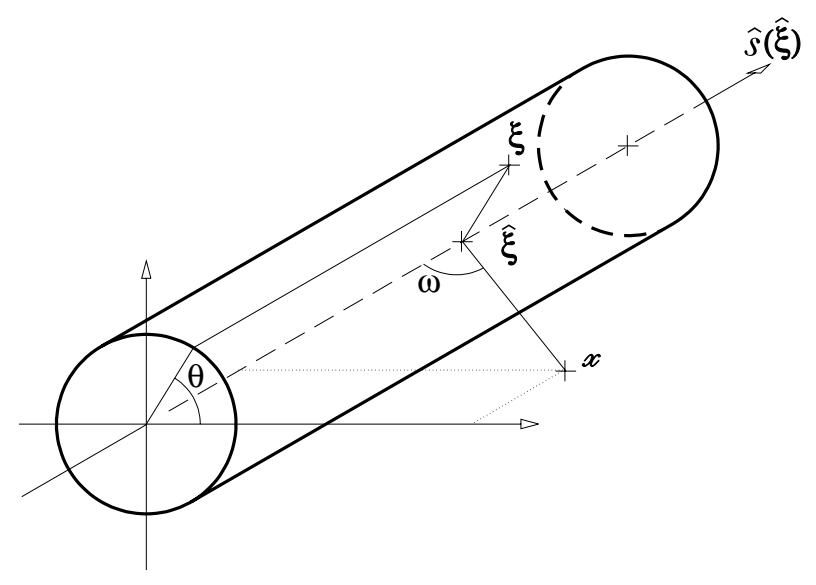

Figure 1.- Analysis of distance between an arbitrary point $\boldsymbol{x}$ and any point $\boldsymbol{\xi}$ at the electrode surface.

as it is shown in figure 1.

The elliptic integral obtained when $r(\boldsymbol{x}, \boldsymbol{\xi})$ in (24) is substituted into (23) can be aproximated by means of numerical integration. In practice, this simplification is quite accurate because we are interested in computing potential at points on the earth surface, which are very far from the earthing electrode in comparison with the size of its diameter. Accordingly, distance between points $\boldsymbol{x}$ and $\hat{\boldsymbol{\xi}}$ is several orders of magnitude bigger than the bar diameter $\phi(\widehat{\boldsymbol{\xi}})[5]$. At the same time, this result can be interpreted as an approximation of distance $r(\boldsymbol{x}, \boldsymbol{\xi})$ in $(24)$, in terms of the distance between $\boldsymbol{x}$ and its orthogonal projection $\widehat{\boldsymbol{\xi}}$ and the cylindrical diameter at this point:

$$
r(\boldsymbol{x}, \boldsymbol{\xi}) \approx \widehat{r}(\boldsymbol{x}, \widehat{\boldsymbol{\xi}})=\sqrt{|\boldsymbol{x}-\widehat{\boldsymbol{\xi}}|^{2}+\frac{\phi^{2}(\widehat{\boldsymbol{\xi}})}{4}}
$$

Finally, analyzing the second term in (23) in the same way as (24), an approximation to the circumferential integral of inner kernel in (18) can be obtained:

$$
\begin{gathered}
\int_{\boldsymbol{\xi} \in C(\widehat{\boldsymbol{\xi}})} k(\boldsymbol{x}, \boldsymbol{\xi}) d C \approx \pi \phi(\widehat{\boldsymbol{\xi}}) \hat{k}(\boldsymbol{x}, \widehat{\boldsymbol{\xi}}), \\
\hat{k}(\boldsymbol{x}, \widehat{\boldsymbol{\xi}})=\left(\frac{1}{\widehat{r}(\boldsymbol{x}, \widehat{\boldsymbol{\xi}})}+\frac{1}{\widehat{r}\left(\boldsymbol{x}, \hat{\boldsymbol{\xi}}^{\prime}\right)}\right) .
\end{gathered}
$$

$\widehat{k}(\boldsymbol{x}, \widehat{\boldsymbol{\xi}})$ is a modified kernel of the original one (4). In this new expression, the orthogonal projection of $\boldsymbol{\xi}$ over the bar axis and the diameter of electrode are used, and distance $r(\boldsymbol{x}, \boldsymbol{\xi})$ is redefined in terms of them. 
On the other hand, taking into account the above analysis of $k(\boldsymbol{x}, \boldsymbol{\xi})$, a first approximation to inner kernel in (15) can now be derived

$$
K(\widehat{\boldsymbol{\chi}}, \widehat{\boldsymbol{\xi}}) \approx \int_{\boldsymbol{\chi} \in C(\widehat{\boldsymbol{\chi}})} \pi \phi(\widehat{\boldsymbol{\xi}}) \hat{k}(\boldsymbol{\chi}, \widehat{\boldsymbol{\xi}}) d C
$$

Next, bearing in mind the hypothesis used in (26), distance between points $\boldsymbol{\chi}$ and $\hat{\boldsymbol{\xi}}$ can be expressed in terms of the distance between points over the axes of electrodes $(\hat{\boldsymbol{\chi}}$ and $\widehat{\boldsymbol{\xi}})$ and the diameter $\phi(\hat{\boldsymbol{\chi}})$, so that kernel (15) can now be simplified in the same manner as (27):

$$
K(\widehat{\boldsymbol{\chi}}, \widehat{\boldsymbol{\xi}}) \approx \pi \phi(\widehat{\boldsymbol{\xi}}) \pi \phi(\widehat{\boldsymbol{\chi}}) \widehat{\hat{k}}(\widehat{\boldsymbol{\chi}}, \widehat{\boldsymbol{\xi}})
$$

being $\widehat{\hat{k}}(\widehat{\boldsymbol{\chi}}, \widehat{\boldsymbol{\xi}})$ the approximated kernel

$$
\begin{gathered}
\hat{\hat{k}}(\hat{\boldsymbol{\chi}}, \widehat{\boldsymbol{\xi}})=\left(\frac{1}{\hat{\widehat{r}}(\hat{\boldsymbol{\chi}}, \widehat{\boldsymbol{\xi}})}+\frac{1}{\widehat{\hat{r}}\left(\widehat{\boldsymbol{\chi}}, \hat{\boldsymbol{\xi}}^{\prime}\right)}\right), \\
\hat{\widehat{r}}(\hat{\boldsymbol{\chi}}, \widehat{\boldsymbol{\xi}})=\sqrt{|\hat{\boldsymbol{\chi}}-\widehat{\boldsymbol{\xi}}|^{2}+\frac{\phi^{2}(\widehat{\boldsymbol{\xi}})+\phi^{2}(\hat{\boldsymbol{\chi}})}{4}} .
\end{gathered}
$$

The use of the unexpensive approximations (27) and (30) to evaluate the circumferential integrals of kernels, takes advantage of the fact that double integration in the general boundary element approach is performed on a 1D domain - expressions (18) and (21)-.

For different selections of the sets of trial and test functions, specific formulations can be obtained. Thus, for constant leakage current elements, Point Colocation (Dirac deltas as trial functions) leads to the very early intuitive methods, such as the superposition of current point sources, whereas Galerkin formulation (test functions identical to trial functions) leads to a kind of more recent methods, such as "Average Potential Method, APM"), based on the idea that each segment of conductor is substituted for a "line of point sources over the length of the conductor" [13]. In these methods, coefficients (21) correspond to "mutual and self resistances" between "segments of conductor" [11]. Naturally, for higher order elements it is now possible to derive more advanced formulations [5]. Further discussion and examples are restricted to Galerkin type formulations, where the matrix of coefficients of linear system (19) is symmetric and positive definite [12].

Now, if we take into account simplifications achieved in the circumferential integration and diameter of conductors is assumed constant within each element, final expressions for computing potential coefficients (18) and linear system coefficients (21) can be written as

$$
\begin{gathered}
\widehat{V}_{i}^{\alpha}(\boldsymbol{x}) \approx \frac{\phi^{\alpha}}{4 \gamma} \int_{\widehat{\boldsymbol{\xi}}_{\in L^{\alpha}}} \hat{k}(\boldsymbol{x}, \widehat{\boldsymbol{\xi}}) \hat{N}_{i}(\widehat{\boldsymbol{\xi}}) d L \\
\widehat{R}_{j i}^{\beta \alpha} \approx \frac{\pi \phi^{\beta} \phi^{\alpha}}{4 \gamma} \int_{\widehat{\boldsymbol{\chi}} \in L^{\beta}} \widehat{N}_{j}(\widehat{\boldsymbol{\chi}})\left[\int_{\hat{\boldsymbol{\xi}}_{\in L^{\alpha}}} \hat{\hat{k}}(\hat{\boldsymbol{\chi}}, \widehat{\boldsymbol{\xi}}) \widehat{N}_{i}(\widehat{\boldsymbol{\xi}}) d L\right] d L,
\end{gathered}
$$


where $\phi^{\alpha}$ and $\phi^{\beta}$ represent the constant diameter within elements $L^{\alpha}$ and $L^{\beta}$. Obviously, (34) leads to a symmetric matrix.

Nevertheless, computation of the remaining integrals in (33) and (34) is not obvious, and the cost of numerical integration is still out of range due to the undesirable behaviour of the integrands. For this reason, it is essential to derive explicit formulae in order to compute analytically these coefficients.

\section{Analytical Integration of Coefficients}

Successive hypotheses introduced in the general boundary element formulation have allowed to reduce the complexity of the grounding grid analysis. Thus, each cylindrical conductor can be modelled by means of a segment of straight line - the electrode axis - defined by its ends, and provided with an additional geometrical property - the electrode diameter - which is taken into account in the calculations.

Now, potential created by an electrode at any point $x$ of the domain (17) can be obtained as sum of the contributions (33) of each conductor of the grounding grid. These terms correspond to the $i$ trial function contribution to potential generated by the element $L^{\alpha}$ belonging to electrode $L$ at an arbitrary point $\boldsymbol{x}$. On the other hand, the simplified 1D boundary element discretization of the problem leads to system (19), which coefficients $\widehat{R}_{j i}^{\beta \alpha}$ in (34) correspond to the $i$ trial function contribution to potential generated by the element $L^{\alpha}$ over other element $L^{\beta}$, weighted by the $j$ test function.

\subsection{Computation of Potential Coefficients $\widehat{V}_{i}^{\alpha}(x)$}

Any point $\widehat{\boldsymbol{\xi}} \in L^{\alpha}$ can be expressed in terms of the mid-point $\widehat{\boldsymbol{\xi}}_{0}$ of the element $L^{\alpha}$, its length $\mathcal{L}^{\alpha}$ and its unit vector $\widehat{\boldsymbol{s}}^{\alpha}$, for a value of scalar parameter $\xi$ varying within the range -1 and 1 (domain of isoparametric trial functions) [14]. Thus, (33) can be rewritten as the line integral in a single variable $\xi$ :

$$
\widehat{V}_{i}^{\alpha}(\boldsymbol{x})=\frac{\phi^{\alpha} \mathcal{L}^{\alpha}}{8 \gamma} \int_{\xi=-1}^{\xi=1} \hat{k}(\boldsymbol{x}, \widehat{\boldsymbol{\xi}}(\xi)) \widehat{N}_{i}(\widehat{\boldsymbol{\xi}}(\xi)) d \xi
$$

In the same way, it is possible to express the integral kernel $\hat{k}(\boldsymbol{x}, \widehat{\boldsymbol{\xi}}(\xi))$ as a function of $\xi$, given that it depends on terms (28) in the form $\widehat{r}(\boldsymbol{x}, \widehat{\boldsymbol{\xi}}(\xi))$. Thus, if we denote $p_{0}$ the distance between the point $\boldsymbol{x}$ and its orthogonal projection over the electrode axial line, and $q$ the distance between this projection and the mid-point $\widehat{\boldsymbol{\xi}}_{0}$, distance $\widehat{r}(\boldsymbol{x}, \widehat{\boldsymbol{\xi}}(\xi))$ results in

$$
\begin{gathered}
\widehat{r}(\boldsymbol{x}, \widehat{\boldsymbol{\xi}}(\xi))=\frac{\mathcal{L}^{\alpha}}{2} \sqrt{(\widehat{p}(\boldsymbol{x}))^{2}+(\widehat{q}(\boldsymbol{x})-\xi)^{2}}, \\
(\widehat{p}(\boldsymbol{x}))^{2}=\left(\frac{p_{0}(\boldsymbol{x})}{\mathcal{L}^{\alpha} / 2}\right)^{2}+\left(\frac{\phi^{\alpha}}{\mathcal{L}^{\alpha}}\right)^{2}, \quad \widehat{q}(\boldsymbol{x})=\frac{q(\boldsymbol{x})}{\mathcal{L}^{\alpha} / 2}
\end{gathered}
$$

Obviously this analysis can also be performed with the term $\widehat{r}\left(\boldsymbol{x}, \hat{\boldsymbol{\xi}}^{\prime}(\xi)\right)$ in $(28)$, and we should obtain analogous expressions in terms of new geometrical parameters $\hat{p}^{\prime}(\boldsymbol{x})$ and $\widehat{q}^{\prime}(\boldsymbol{x})$, corresponding to points $\left(x, \hat{\boldsymbol{\xi}}^{\prime}\right)[5]$. 
On the other hand, trial functions $\widehat{N}_{i}(\widehat{\xi}(\xi))$ in (35) can be expressed - by means of their series expansion until the second order term - as parabolic functions in the variable $\xi$, which coefficients depend on known values of the functions and their first and second derivatives [5].

Finally, if we substitute in (35) expressions obtained in (36) for the integral kernel (28) and those developed for the trial functions $\widehat{N}_{i}(\widehat{\boldsymbol{\xi}}(\xi)$ ), taking into account that both depend on $\xi$, it is possible to integrate explicitly the potential coefficient $\widehat{V}_{i}^{\alpha}(\boldsymbol{x})$. After a relatively long analytical development, (35) results in

$$
\widehat{V}_{i}^{\alpha}(\boldsymbol{x})=\frac{\phi^{\alpha}}{4 \gamma}\left[\Phi(\widehat{p}(\boldsymbol{x}), \widehat{q}(\boldsymbol{x}))+\Phi\left(\hat{p}^{\prime}(\boldsymbol{x}), \widehat{q}^{\prime}(\boldsymbol{x})\right)\right]
$$

where function $\Phi(\cdot, \cdot)$ depends only on geometrical parameters and known coefficients of trial functions [5].

\subsection{Computation of System Coefficients $\widehat{R}_{j i}^{\beta \alpha}$}

In analogous way to previous development, any point $\widehat{\boldsymbol{\chi}} \in L^{\beta}$ can be expressed in terms of the mid-point $\widehat{\boldsymbol{\chi}}_{0}$ of the element $L^{\beta}$, its length $\mathcal{L}^{\beta}$ and its unit vector $\widehat{\boldsymbol{s}}^{\beta}$, for a value of scalar parameter $\chi$ varying within the range -1 and 1 (domain of isoparametric trial functions) [14]. Thus, taking into account the development achieved in (35), expression (34) can be rewritten as two line integrals, one in the single variable $\xi$ and other in $\chi$,

$$
\widehat{R}_{j i}^{\beta \alpha}=\frac{\pi \phi^{\alpha} \phi^{\beta} \mathcal{L}^{\alpha} \mathcal{L}^{\beta}}{16 \gamma}\left\{\int_{\chi=-1}^{\chi=1} \widehat{N}_{j}(\widehat{\boldsymbol{\chi}}(\chi))\left[\int_{\xi=-1}^{\xi=1} \widehat{\hat{k}}(\widehat{\boldsymbol{\chi}}(\chi), \widehat{\boldsymbol{\xi}}(\xi)) \widehat{N}_{i}(\widehat{\boldsymbol{\xi}}(\xi)) d \xi\right] d \chi\right\}
$$

It may be seen that the line integral in $\xi$ is similar to (35), although in this case, the integral kernel is given by (31). If geometrical parameters $\widehat{p}(\widehat{\chi}(\chi))$ and $\widehat{q}(\widehat{\chi}(\chi))$ are suitably redefined, expression (35) can be written [5] - by means of (38) - in the form

$$
\widehat{R}_{j i}^{\beta \alpha}=\frac{\pi \phi^{\alpha} \phi^{\beta} \mathcal{L}^{\beta}}{8 \gamma}\left\{\widehat{\mathcal{R}}_{j i}^{\beta \alpha}\left(\widehat{\boldsymbol{\xi}}_{1}, \widehat{\boldsymbol{\xi}}_{2}, \widehat{\boldsymbol{\chi}}_{1}, \widehat{\boldsymbol{\chi}}_{2}\right)+\widehat{\mathcal{R}}_{j i}^{\beta \alpha}\left(\widehat{\boldsymbol{\xi}}_{1}^{\prime}, \widehat{\boldsymbol{\xi}}_{2}^{\prime}, \widehat{\boldsymbol{\chi}}_{1}, \widehat{\boldsymbol{\chi}}_{2}\right)\right\}
$$

where coefficients $\widehat{\mathcal{R}}_{j i}^{\beta \alpha}\left(\widehat{\boldsymbol{\xi}}_{1}, \widehat{\boldsymbol{\xi}}_{2}, \widehat{\boldsymbol{\chi}}_{1}, \widehat{\boldsymbol{\chi}}_{2}\right)$ can be obtained as

$$
\begin{gathered}
\widehat{\mathcal{R}}_{j i}^{\beta \alpha}\left(\hat{\boldsymbol{\xi}}_{1}, \widehat{\boldsymbol{\xi}}_{2}, \hat{\boldsymbol{\chi}}_{1}, \hat{\boldsymbol{\chi}}_{2}\right)=\int_{\chi=-1}^{\chi=1} \widehat{N}_{j}(\widehat{\boldsymbol{\chi}}(\chi))[\Phi(\widehat{p}(\widehat{\boldsymbol{\chi}}(\chi)), \widehat{\boldsymbol{q}}(\widehat{\boldsymbol{\chi}}(\chi)))] d \chi \\
\hat{p}^{2}(\widehat{\boldsymbol{\chi}}(\chi))=\left(\frac{p_{0}(\hat{\boldsymbol{\chi}}(\chi))}{\mathcal{L}^{\alpha} / 2}\right)^{2}+\left(\frac{\phi^{\alpha}}{\mathcal{L}^{\alpha}}\right)^{2}+\left(\frac{\phi^{\beta}}{\mathcal{L}^{\alpha}}\right)^{2}, \\
\widehat{q}(\widehat{\boldsymbol{\chi}}(\chi))=\frac{q(\widehat{\boldsymbol{\chi}}(\chi))}{\mathcal{L}^{\alpha} / 2} .
\end{gathered}
$$

On the other hand, trial functions $\widehat{N}_{j}(\widehat{\chi}(\chi))$ can be expressed - by means of their series expansion until the second order term - as parabolic functions in the variable $\chi$, which coefficients are known [5], in the same way as it has been previously made with $\widehat{N}_{i}(\widehat{\xi}(\xi))$. Finally, substitution of trial functions $\widehat{N}_{j}(\widehat{\boldsymbol{\chi}}(\chi))$ in (41) leads to a line integral in the variable $\chi$. 


\subsubsection{Integration of Coefficients $\widehat{\mathcal{R}}_{j i}^{\beta \alpha}\left(\widehat{\boldsymbol{\xi}}_{1}, \widehat{\boldsymbol{\xi}}_{2}, \widehat{\boldsymbol{\chi}}_{1}, \widehat{\boldsymbol{\chi}}_{2}\right)$}

Each coefficient $\widehat{\mathcal{R}}_{j i}^{\beta \alpha}\left(\widehat{\boldsymbol{\xi}}_{1}, \widehat{\boldsymbol{\xi}}_{2}, \widehat{\boldsymbol{\chi}}_{1}, \widehat{\boldsymbol{\chi}}_{2}\right)$ in $(40)$ can be understood as the potential influence generated by an electrode on another. Since electrodes are perfectly defined by cartesian coordinates of their axial ends, we can analyse the first of two terms and apply results and formulae obtained to the second one, considered as the integration between two different bars (with the symmetric points to $\widehat{\boldsymbol{\xi}}_{1}$ and $\widehat{\boldsymbol{\xi}}_{2}$ ).

Therefore, integration of coefficients (41) requires in the first place a geometrical analysis of two cylindrical bars in the space. This study allows to express adimensional distances $\widehat{p}(\widehat{\boldsymbol{\chi}}(\chi))$ and $\widehat{q}(\widehat{\boldsymbol{\chi}}(\chi))$ in $(42)$ and (43) as a function of $\chi$, and a set of known geometrical parameters depending on the relative position between electrodes [5]. Now, if final expressions for $\widehat{p}(\widehat{\boldsymbol{\chi}}(\chi))$ and $\widehat{q}(\widehat{\chi}(\chi))$ derived with the previous analysis, and those obtained for trial functions $\hat{N}_{j}(\widehat{\boldsymbol{\chi}}(\chi))$ are substituted in (41), and we make suitable arrangements, results in

$$
\widehat{\mathcal{R}}_{j i}^{\beta \alpha}\left(\widehat{\boldsymbol{\xi}}_{1}, \widehat{\boldsymbol{\xi}}_{2}, \widehat{\boldsymbol{\chi}}_{1}, \widehat{\boldsymbol{\chi}}_{2}\right)=\sum_{u=0}^{u=2} \sum_{w=0}^{w=4} \mathcal{K}_{w}^{(u)} \varphi_{w}^{(u)}
$$

where coefficients $\mathcal{K}_{w}^{(u)}$ can directly be computed from the $j$ th trial function, the geometrical parameters of electrodes and the $i$ th trial function [5].

On the other hand, remaining line integrals in the variable $\chi$ are incorporated in coefficients $\varphi_{w}^{(u)}(44)$. Development of explicit formulae to evaluate these expressions is not obvious, and requires quite a lot of analytical work. Moreover, this circumstance gets worse because coefficients $\varphi_{w}^{(u)}$ depends also on the geometrical parameters of electrodes, which possible values increase the number of cases of different types of integrals we must analyse, due to singularities that can be produced [5].

For this reason, in the beginning of this project [15] analytical expressions for the more common spatial arrangements of electrodes - perpendicular and parallel bars - were derived. Although these techniques represented a significant improvement in the area of earthing analysis, it was necessary to complete the analysis of integrals independently of geometrical parameters, in order to compute them analytically in all cases.

At present, this development has been completely finished, and now we get ready explicit expressions

to compute all coefficients $\varphi_{w}^{(u)}$, although its derivation is too cumbersome to be made explicit in this paper [5]. These formulae have been developed in order to make easy the later implementation in a computer code, in such a way as its evaluation is made in recurrent form, using as few as possible operations with transcendental functions. Nevertheless, its programming must be done carefully, due to the huge complexity of the final formulae of coefficients in (44), and its ill-conditioning.

\section{Application to a Real Case}

This simplified 1D numerical approach based on the Boundary Element Method with analytical integration of coefficients of the linear equations system, is very structured, and it has been developed to be implemented in a Computer Aided Design system. 


\begin{tabular}{|c|c|}
\hline \multicolumn{2}{|c|}{ E. R. BARBERÁ GROUNDING SYSTEM } \\
\hline Max. Grid Dimensions: & $145 \mathrm{~m} \times 90 \mathrm{~m}$ \\
Total Protected Surface: & $6500 \mathrm{~m}^{2}$ \\
Grid Depth: & $0.80 \mathrm{~m}$ \\
Number of Grid Electrodes: & 408 \\
Max./Min. Electrode Length: & $19 \mathrm{~m} / 3 \mathrm{~m}$ \\
Electrode Diameter: & $12.85 \mathrm{~mm}$ \\
Ground Potential Rise: & $10 \mathrm{kV}$ \\
Earth Resistivity: & $60 \Omega \mathrm{m}$ \\
\hline
\end{tabular}

Table 1.-E. R. Barberá Substation: Characteristics.

Nowadays, all these techniques derived by the authors have allowed to develop the system TOTBEM for the computer design of earthing grids of electrical substations [16]. With this system, now it is possible to analyse accurately grounding grids of huge installations, with acceptable computing requirements in memory storage and CPU time.

The example that we present is the E. R. Barberá substation grounding, close to Barcelona, Spain. The earthing system of this substation is a grid of 408 cylindrical conductors with constant diameter $(12.85 \mathrm{~mm})$ buried to a depth of $80 \mathrm{~cm}$, being the total surface protected up to $6500 \mathrm{~m}^{2}$. The total area studied is a rectangle of $135 \mathrm{~m}$ by $210 \mathrm{~m}$, which implies a surface up to $28000 \mathrm{~m}^{2}$. The plan of the grounding grid and its characteristics are presented in figure 2. a) and table 1.

The numerical model used in the resolution of this problem has been a Galerkin formulation. Each bar is discretized in one single constant leakage current density element, which implies 408 degrees of freedom. On the other hand, the ground potential rise considered in this study has been $10 \mathrm{kV}$ (due to the linear relation between potential and intensity, we can indistinctly consider the Ground Potential Rise or the Total Surge Current).

Numerical results, such as the total fault current and the equivalent resistance of the grounding system, are given in table 2. Moreover, figure 2. b) shows the potential distribution on ground surface when fault condition ocurrs, figure 2. c) represents the potential profile along a line, and figure 2. d) is a $3 \mathrm{D}$ view of potential level on surface. This numerical model of the grounding grid has only required seven and a half minutes of CPU time in a conventional personal computer (i.e. PC486/16Mb to $66 \mathrm{MHz}$ ). It is obviuos that this proposed approach allows the complete characterization of a grounding grid in a riguorous and reliable way, with very acceptable computing requirements.

This example has also been solved increasing the number of boundary elements used in the numerical model, by means of the subdivision of each one of the electrodes of the grid. At the scale of the whole grid, results and potential distributions are not noticeably improved by increasing discretization, therefore as a general rule, it will not be considered necessary the additional subdivision of grid conductors. In cases in 
which we need more accurate results, i.e. to compute touch or step voltages [1], the use of higher order elements (linear or parabolic) are more advantageous in comparison with constant elements [5].

\begin{tabular}{|c|c|}
\hline \multicolumn{2}{|c|}{ E. R. BARBERÁ GROUNDING SYSTEM: } \\
1D BEM MODEL \& RESULTS \\
\hline Type of Element: & Constant \\
Number of Nodes: & 238 \\
Number of Elements: & 408 \\
\hline Fault Current: & $31.75 \mathrm{kA}$ \\
Equivalent Resistance: & $0.315 \Omega$ \\
CPU Time: & $450 \mathrm{~s}$ \\
Computer: & PC $486 / 66 \mathrm{MHz}$ \\
\hline
\end{tabular}

Table 2.-E.R. Barberá Substation: Numerical Model and BEM Results.

\section{Conclusions}

A Boundary Element approach for the numerical computation of substation grounding systems developed by the authors in the last years has been presented. For 3D problems, some reasonable assumptions allow to reduce the general 2D BEM formulation to an approximated less expensive 1D version. Efforts have been particularly made in getting a drastical reduction in computing time by means of new completely analytical integration techniques, while semi-iterative methods have proved to be specially efficient for solving the involved system of linear equations.

On the other hand, several widespread intuitive methods (such as the Average Potential Method) can be identified in this general formulation as the result of suitable assumptions introduced in the BEM formulation to reduce computational cost for specific choices of the test and trial functions. Problems encountered by other authors with the application of these methods can now be mathematically explained and sources of error pointed out, while more efficient and accurate formulations can now be derived.

The numerical approach proposed is a general methodology that - for the first time - allows to obtain high accuracy results in the grounding grid analysis of electrical substations of medium/big sizes, using a low cost and widely available conventional computer. Obviously, study of big installations should require higher computing efforts with more powerful computers, although always with a very reasonable cost.

\section{Acknowledgments}

This work has been partially supported by the power company "Unión Fenosa", by research fellowships of the R\&D General Secretary of the "Xunta de Galicia" and the University of La Coruña, and by the company "Fecsa". 


\section{References}

[1] Sverak J.G., Dick W.K., Dodds T.H., Heppe R.H., 1981, "Safe substation grounding. (Part I)", IEEE Trans. on Power App. and Sys., 100, 4281-90.

[2] Sverak J.G. et al., 1982, "Safe substation grounding. (Part II)", IEEE Trans. on Power App. and Sys., 101, 4006-23.

[3] Durand, 1966, "Électrostatique", Masson, Paris.

[4] Navarrina F., Colominas I., Casteleiro M., 1992, "Analytical integration techniques for the earthing grid computation by boundary element methods", International Congress on Numerical Methods in Engineering and Applied Sciences, II, 1197-1206. Concepción, (Chile).

[5] Colominas I., 1995, "Cálculo y Diseño por Ordenador de Tomas de Tierra en Instalaciones Eléctricas: Una Formulación Numérica basada en el Método Integral de Elementos de Contorno", Ph. D. Thesis, E.T.S. de Ingenieros de Caminos, Canales y Puertos. Universidad de La Coruña (Spain).

[6] Heppe R.J., 1979, "Computation of potential at surface above an energized grid or other electrode, allowing for non-uniform current distribution", IEEE Trans. on Power App. and Sys., 98, 1978-88.

[7] Beskos D.E., 1987, "Potential Theory", Boundary Element Methods in Mechanics, 23-106, D.E. Beskos (Editor), Noth-Holland, Amsterdam.

[8] Brebbia C.A., Domínguez J., 1989, "Boundary Elements: An Introductory Course", Computational Mechanics Publications, Southampton.

[9] Lean M.H. et al., 1979, "Application of the BEM in Electrical Engineering Problems", Developments in BEM's-I, 207-250, Banerjee P.K and Butterfield R. (Editors), Applied Science, London.

[10] Stakgold I., 1979, "Green's functions and boundary value problems", Wiley. New York.

[11] Garrett D.L., Pruitt J.G., 1985, "Problems encountered with Average Potential Method of analyzing substation grounding systems", IEEE Trans. on Power App. and Sys., 104, 3586-96.

[12] Johnson C., 1987, "Numerical solution of partial differential equations by the finite element method", Cambridge Univ. Press, Cambridge (USA).

[13] Navarrina F., Moreno L., Bendito E., Encinas A., Ledesma A., Casteleiro M., 1991, "Computer aided design of grounding grids: a boundary element approach", Mathematics in Industry, 307-14, Kluwer Academic Pub., Dordrecht, (Netherlands).

[14] Hughes T.J.R., 1987, "The finite element method", Prentice Hall. New Jersey.

[15] Colominas I., Navarrina F., Casteleiro M., 1993, "Fórmulas analíticas de integración para el cálculo de tomas de tierra mediante el método de los elementos de contorno", II Congreso de Métodos Numéricos en Ingeniería, II, 857-64. La Coruña.

[16] Casteleiro M., Hernández L.A., Colominas I., Navarrina F., 1994, "Memoria y Manual de Usuario del Sistema TOTBEM para Cálculo y Diseño Asistido por Ordenador de Tomas de Tierra de Instalaciones Eléctricas", E.T.S. de Ingenieros de Caminos, C. y P. Universidad de La Coruña (Spain). 


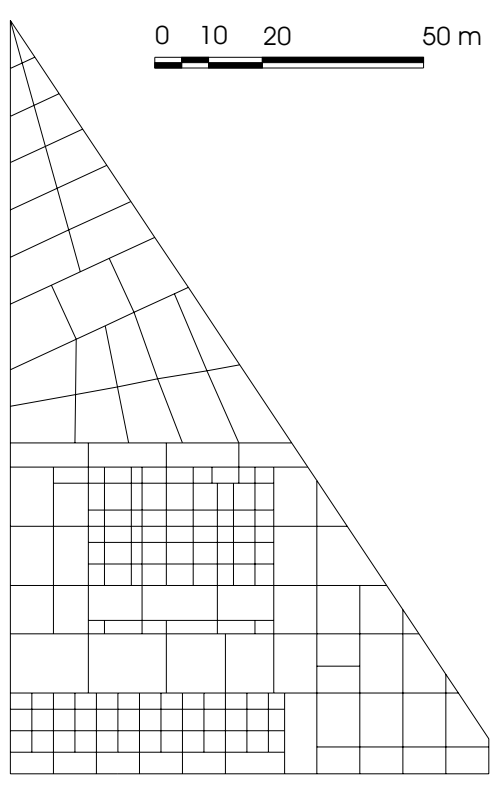

a)
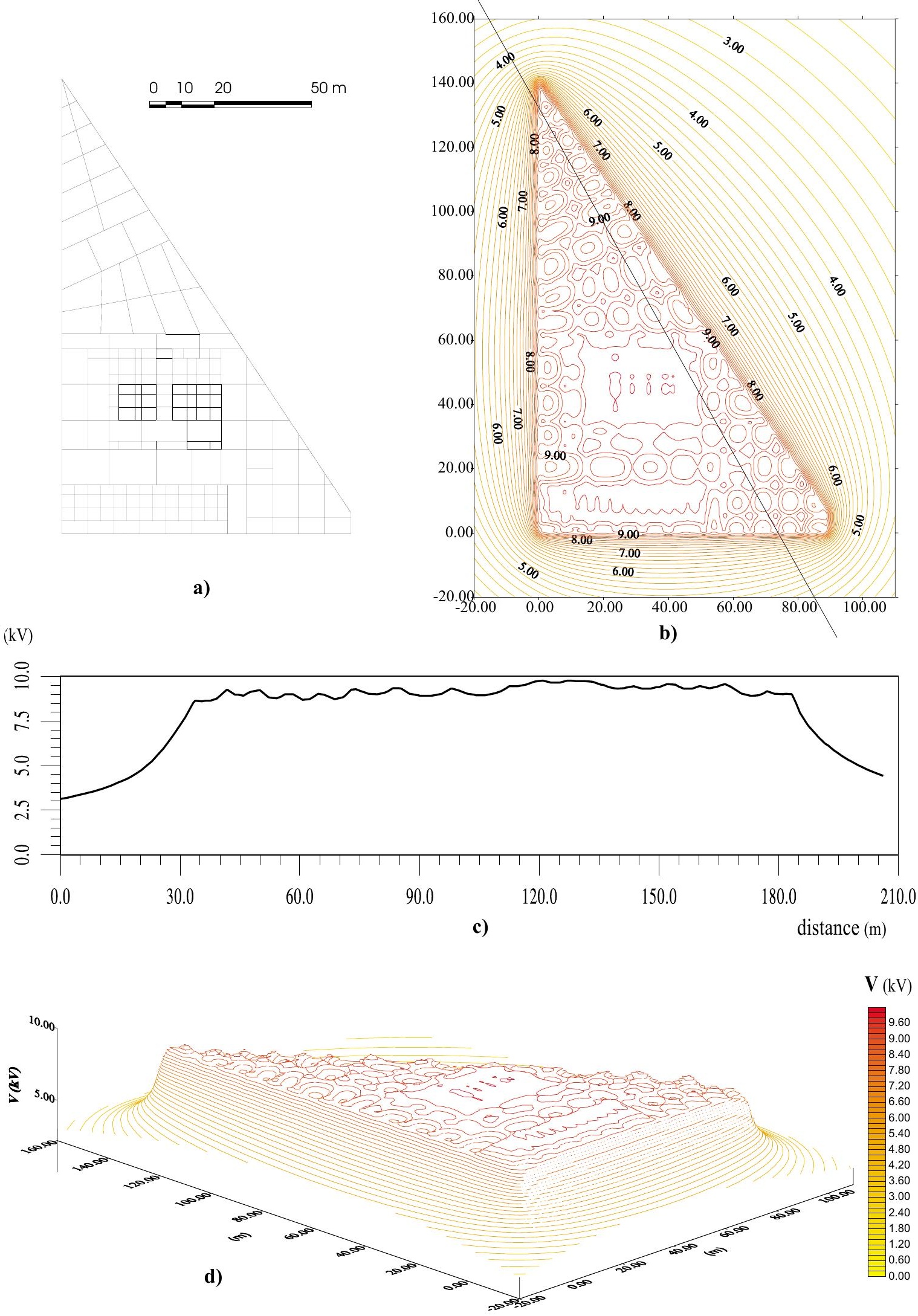

Figure 2.-- E. R. Barberá Substation: a) Plan of the grounding grid, b) Potential distribution on ground surface $(\mathrm{kV})$, c) Potential profile along a line, d) 3D view of potential level on ground surface. 